\section{Cetuximab in der Erstlinie nicht erfolgreich}

Patienten mit kolorektalem Karzinom (CRC) profitieren von Agenzien gegen den epidermalen Wachstumsfaktor-Rezeptor (EGFR). In einer aktuellen Studie konnte der Anti-EGFR-Antikörper Cetuximab in Kombination mit einer Oxaliplatin-haltigen Chemotherapie das Überleben jedoch nicht verlängern.

A ntikörper gegen die extrazelluläre EGFR-Domäne haben beim CRC bereits einen klinischen Vorteil gezeigt - bei Patienten ohne Mutation im K-RasGen. Nun liegen die Daten einer PhaseIII-Studie des britischen Medical Research Council vor, in der Cetuximab mit dem Standard-Regime für die Erstlinien-Chemotherapie des fortgeschrittenen kolorektalen Karzinoms kombiniert wurde.

Die Patienten wurden in drei Gruppen randomisiert: Arm A erhielt Oxaliplatin und ein Fluoropyrimidin, Arm B dieselbe Kombination plus Cetuximab und Arm C eine intermittierende Chemotherapie. Die Wahl des Fluoropyrimidins (Capecitabin oder eine Infusion von Fluorouracil plus Folinsäure) wurde vor der Randomisierung getroffen.

\section{Auf Knochen blicken!}

In einer retrospektiven Studie wurde der Krankheitsverlauf bei 264 Patienten mit Knochenmetastasen eines Kolorektalkarzinoms beobachtet.

A uf je zehn Darmkrebs-Patienten kommt etwa einer mit Knochenmetastasen. Insgesamt hatten vier von fünf dieser Patienten bei der Krebsdiagnose in Arm A auf 64\% im Cetuximab-Arm. testinaltrakts (97 versus 67 Patienten). kam es bei $10 \%$ dieser Patienten.
In der hier vorgestellten Publikation wurden Arm A und B verglichen. $43 \%$ der 1.316 Patienten, für die dazu Daten vorlagen, hatten $K$-Ras-Mutationen im Tumor. Unter den Patienten mit K-RasWildtyp (367 in Arm A und 362 in Arm B) unterschied sich das Gesamtüberleben nicht zwischen den Behandlungsarmen. Es lag bei 17,9 Monaten in der Kontrollgruppe und bei 17,0 Monaten in der Cetuximab-Gruppe. Auch gab es keinen Effekt auf das progressionsfreie Überleben (8,6 Monate in beiden Gruppen). Die Gesamtansprechrate erhöhte sich von $57 \%$

Unter dem Antikörper kam es häufiger zu schweren Nebenwirkungen der Haut (114 vs. 14 Patienten) und des Gastroin-

einen fortgeschrittenen Tumor im Stadium T3/4. Hauptort der Metastasen war die Wirbelsäule (65\%), gefolgt von Hüfte und Becken (34\%) sowie den Extremitätenknochen (26\%). Median waren elf Monate nach der Diagnose die ersten Knochenmetastasen nachweisbar, zwei Monate später auch erste Komplikationen wie Frakturen, Rückenmarkskompressionen oder Bestrahlungen. Zu Frakturen

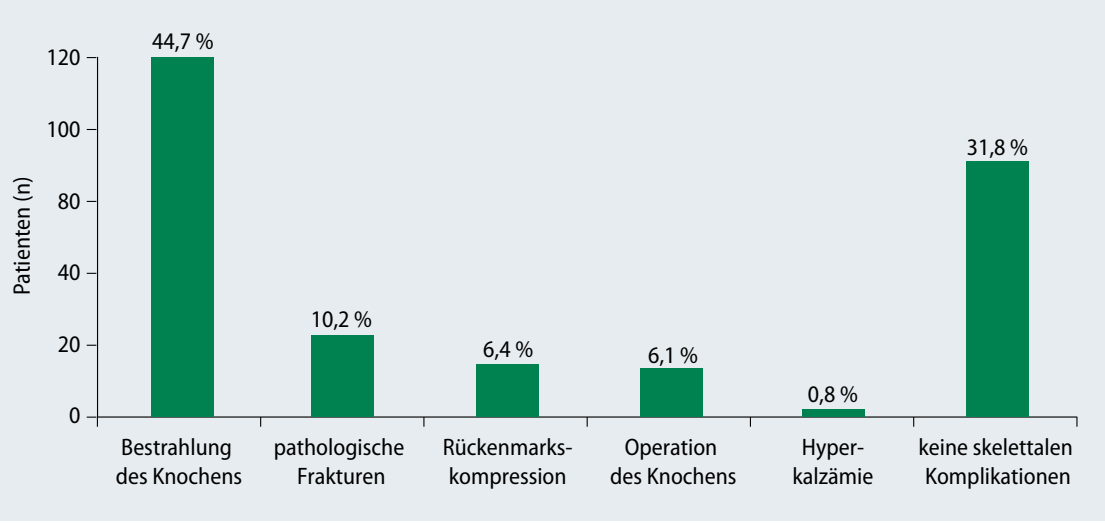

Abb.: Inzidenz skelettaler Komplikationen in Patienten mit Knochenmetastasen aufgrund eines Kolorektalkarzinoms $(n=264)$, unabhängig von einer Therapie
Das Gesamtüberleben von Patienten mit verschiedenen somatischen Mutationen unterschied sich unabhängig von der erhaltenen Behandlung: Patienten mit $B$-Raf-Mutation lebten im Durchschnitt 8,8 Monate; solche mit K-Ras-Mutation 14,4 Monate und Patienten mit reinen Wildtyp-Tumoren 20,1 Monate.

Fazit: In dieser groß angelegten PhaseIII-Studie konnte nicht bestätigt werden, dass die Zugabe von Cetuximab zu einer Oxaliplatin-basierten Erstlinien-Chemotherapie Patienten mit fortgeschrittenem kolorektalen Karzinom nützt. Zwar erhöhte der Antikörper die Ansprechrate, verlängerte aber nicht das Überleben auch nicht bei Patienten mit K-Ras-Wildtyp.

Christina Berndt

Maughan TS et al. Addition of cetuximab to oxaliplatin-based first-line combination chemotherapy for treatment of advanced colorectal cancer: results of the randomised phase 3 MRC COIN trial. Lancet. 2011; 377(9783):2103-14.
$48 \%$ der Patienten erhielten Zoledronsäure. Dadurch ließen sich Skelettkomplikationen signifikant um etwa einen Monat verzögern. Die mediane Überlebenszeit nach der Diagnose von Knochenmetastasen lag bei sieben Monaten, unabhängig davon, ob Skelettkomplikationen auftraten oder mit Zoledronsäure behandelt worden war. Bei den 157 Patienten mit Daten zu einer Bisphosphonat-Therapie betrug die Überlebenszeit mit Zoledronsäure median zehn, ohne das Medikament nur sechs Monate $(\mathrm{p}=0,161)$.

Fazit: Knochenmetastasen reduzieren die Überlebenszeit von Patienten mit metastasiertem kolorektalem Karzinom von im Schnitt über 20 Monate um zwei Drittel sowie auf etwa vier bis fünf Monate, wenn es zu Skelettkomplikationen kommt. Eine rechtzeitige Therapie kann diese Komplikationen verzögern und das Leben verlängern.

Thomas Müller

Santini D et al. Natural history of bone metastasis in colorectal cancer: final results of a large Italian bone metastases study. Ann Oncol. 2012 Feb 7. [Epub ahead of print]. 